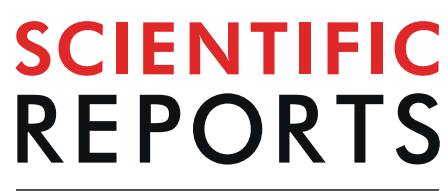

natureresearch

Check for updates

\title{
Asymmetric electrode incorporated 2D GeSe for self-biased and efficient photodetection
}

Muhammad Hussain ${ }^{1}$, Sikandar Aftab ${ }^{2}$, Syed Hassan Abbas Jaffery ${ }^{1}$, Asif Ali ${ }^{1}$, Sajjad Hussain ${ }^{1}$ Dinh Nguyen Cong ${ }^{1}$, Raheel Akhtar ${ }^{3}$, Yongho Seo ${ }^{1}$, Jonghwa Eom ${ }^{2}$, Praveen Gautam², Hwayong Noh ${ }^{2}$ \& Jongwan Jung ${ }^{1 凶}$

2D layered germanium selenide (GeSe) with $p$-type conductivity is incorporated with asymmetric contact electrode of chromium/Gold ( $\mathrm{Cr} / \mathrm{Au}$ ) and Palladium/Gold (Pd/Au) to design a self-biased, high speed and an efficient photodetector. The photoresponse under photovoltaic effect is investigated for the wavelengths of light (i.e. $\sim 220, \sim 530$ and $\sim 850 \mathrm{~nm}$ ). The device exhibited promising figures of merit required for efficient photodetection, specifically the Schottky barrier diode is highly sensitive to NIR light irradiation at zero voltage with good reproducibility, which is promising for the emergency application of fire detection and night vision. The high responsivity, detectivity, normalized photocurrent to dark current ratio (NPDR), noise equivalent power (NEP) and response time for illumination of light $(\sim 850 \mathrm{~nm})$ are calculated to be $280 \mathrm{~mA} / \mathrm{W}, 4.1 \times 10^{9}$ Jones, $3 \times 10^{7} \mathrm{~W}^{-1}, 9.1 \times 10^{-12}$ $\mathrm{WHz}^{-1 / 2}$ and $69 \mathrm{~ms}$ respectively. The obtained results suggested that $\mathrm{p}-\mathrm{GeSe}$ is a novel candidate for SBD optoelectronics-based technologies.

Two-dimensional (2D) materials have chronically been the most widely studied materials, particularly after the successful scotch tape test to exfoliate graphene by Andre Geim and Kostya Novoselov in 2004 ${ }^{1}$. 2D materials possess excellent electrical and mechanical properties toward diverse electronic device applications. Graphene, being the prototype of $2 \mathrm{D}$ materials ${ }^{2,3}$, has been studied broadly for its exotic electrical, optical, and mechanical properties $^{3,4}$. Besides, the group- $I V$ transition metal dichalcogenides (TMDs) having a bandgap of around 1 to $2 \mathrm{eV}^{5-7}$ have attracted increasing interest because of their promising electronic and optoelectronic device applications ${ }^{3,8-21}$. Graphene possesses extremely high carrier mobility $\left(>10^{5} \mathrm{~cm}^{2} \mathrm{~V}^{-1} \mathrm{~s}^{-1}\right)$, but the absence of band gap limits its electronic and optoelectronic applications ${ }^{22}$. Therefore, TMDs with the properties of graphene-like stature, bandgap tunability, weak van der Waals-like forces and stability have intrigued the interest of the scientific community. TMDs are the family of $2 \mathrm{D}$ materials having the chemical composition of $\mathrm{MX}_{2}$, where $\mathrm{M}$ stands for the transition metal elements $(\mathrm{M}=\mathrm{Mo}, \mathrm{W}, \mathrm{Ta}, \mathrm{Ge} . . \mathrm{etc})$ and $\mathrm{X}$ for the chalcogen elements $(\mathrm{X}=\mathrm{Se}$, $\mathrm{S}$ and $\mathrm{Te}$ ). Among TMDs, Ge-based materials are preferred for applications due to their abundance on earth and environmentally friendly nature ${ }^{23}$. With Se, the $p$-type Germanium from a narrow bandgap semiconductor material as $p$-GeSe having exciting application in near-infrared (NIR) photodetectors and electron tunnelling devices. $p$-GeSe has an indirect bandgap of $1.08 \mathrm{eV}$ in the bulk ${ }^{24,25}$, and a direct bandgap of $\sim 1.7 \mathrm{eV}$ in monolayers $^{24,26,27}$. Few layers of $p$-GeSe can be obtained from bulk by mechanical exfoliation method ${ }^{28}$. Among the many applications, p-GeSe shows tremendous capability in the realm of photovoltaics, because of its excellent optical, material and electrical properties. Therefore, it is well known as substitution of phosphorene ${ }^{29}$. Moreover, $\mathrm{GeSe}$ is considered as an ambient stable material ${ }^{30,31}$. Single crystal $p$-GeSe has hole mobilities of $95 \mathrm{~cm}^{2} \mathrm{~V}^{-1} \mathrm{~s}^{-1}$ at $300 \mathrm{~K}$ and $663 \mathrm{~cm}^{2} \mathrm{~V}^{-1} \mathrm{~s}^{-1}$ at $112 \mathrm{~K}^{24,32}$. Theoretical elucidations predicted that the average hole mobility for monolayer GeSe is as high as $1.1 \times 10^{3} \mathrm{~cm}^{2} \mathrm{~V}^{-1} \mathrm{~s}^{-1}$ at $300 \mathrm{~K}^{24}$. Meanwhile, Metal/TMD interface plays an active role in enhancing photoresponse in photodetection applications ${ }^{33-35}$. Therefore, it is important to understand the nature of the Metal/TMD interface in device applications. Recently, 2D heterostructure has been extensively studied for an ideal platform for diode devices, e.g., GaSe/MoSe ${ }_{2}{ }^{36}, \mathrm{MoS}_{2} / \mathrm{Black}_{\text {Phosphorus }}{ }^{37}, \mathrm{MoS}_{2} / \mathrm{p}-\mathrm{Si}$ junction ${ }^{38}$, $\mathrm{WSe}_{2} / \mathrm{MoS}_{2}$ heterojunction ${ }^{39}$ and p-type $\mathrm{MoSe}_{2}$ via Nb-elemental doping ${ }^{40}$ etc. Despite thorough research on bulk

${ }^{1}$ Department of Nanotechnology and Advanced Materials Engineering, and HMC, Sejong University, Seoul, 05006, South Korea. ${ }^{2}$ Department of Physics \& Astronomy and Graphene Research Institute-Texas Photonics Center International Research Center (GRI-TPC IRC), Sejong University, Seoul, 05006, Korea. ${ }^{3}$ Department of Electrical Engineering University of Lahore, Islamabad, Pakistan. ${ }^{\bowtie}$-mail: jwjung@sejong.ac.kr 
GeSe crystals and numerous theoretical calculations on monolayers ${ }^{24,26,41}$, to the best of our knowledge, by using $\mathrm{p}-\mathrm{GeSe}$ on $(\mathrm{Cr} / \mathrm{Au})$ and $(\mathrm{Pd} / \mathrm{Au})$ contact, no experimental investigations have focused on Schottky-Barrier diode (SBD) by asymmetric metal contact with NIR wavelength excitation.

The metal-semiconductor (MS) junction is well known to be a Schottky barrier or ohmic junction based on the corresponding work function of metal compared to the semiconductor and its conductivity type. For the p-type semiconductor, the Schottky barrier is formed when metal work function is smaller than the semiconductor ${ }^{5,42-44}$. SBD formed by Schottky barrier is a widely used component in electronics such as multipliers, microwave mixer and photodetectors, due to its high-frequency capability ${ }^{45}$ and strong nonlinear current-voltage characteristics. Moreover, it is known that SBDs show promising electronic applications and can be used extensively in power electronics due to the low voltage drop in forward bias. It can also be used as photodiodes, power diodes, sensors, varistors and varactors owing to the nonlinear $I-V$ behavior ${ }^{5,46}$.

In this research work, the p-GeSe based FETs were fabricated by using two metals contacts $(\mathrm{Cr} / \mathrm{Au})$ and $(\mathrm{Pd} /$ $\mathrm{Au}$ ) to determine the charge carrier transport and the interface characteristics at the junction. The hole mobility of p-GeSe with $\mathrm{Pd} / \mathrm{Au}$ electrodes was $\sim 5.0 \mathrm{~cm}^{2} \mathrm{~V}^{-1} \mathrm{~s}^{-1}$. Notably, $p$-GeSe is in ohmic contact with $(\mathrm{Pd} / \mathrm{Au})$ while having a Schottky barrier with $(\mathrm{Cr} / \mathrm{Au})$. SBD was made from asymmetric electrodes of $(\mathrm{Pd} / \mathrm{Au})$ and $(\mathrm{Cr} / \mathrm{Au})$. We found the rectifying behavior with an on/off ratio of $\sim 10^{3}$. Besides, we also have extensive studies of the photoconductivity of all fabricated devices under different laser illumination with a wavelength from $220 \mathrm{~nm}$ to $850 \mathrm{~nm}$. It is observed that the SBD is highly sensitive to NIR light irradiation at zero voltage with good reproducibility. The responsivity, detectivity, normalized photocurrent to dark current ratio (NPDR), noise equivalent power $(\mathrm{NEP})$ and response time are estimated to be $220 \mathrm{~mA} / \mathrm{W}, 4.1 \times 10^{9}$ Jones, $3 \times 10^{7} \mathrm{~W}^{-1}, 9.1 \times 10^{-12} \mathrm{WHz}^{1 / 2}$ and $69 \mathrm{~ms}$, respectively. There were a few reports in self-powered SBD using $2 \mathrm{D}$ materials such as $\mathrm{MoS}_{2}, \mathrm{WS}_{2}, \mathrm{InSe}_{\text {, }}$ and BN,but NIR detection using $2 \mathrm{D}$ GeSe has not reported yet. The obtained results suggest that p-GeSe is a novel candidate for SBD optoelectronics-based technologies.

\section{Results and Discussion}

Figure 1a illustrates the schematic diagram of the as-fabricated device having both Schottky and ohmic junction due to asymmetric contact electrodes $(\mathrm{Pd} / \mathrm{Au}$ and $\mathrm{Cr} / \mathrm{Au})$ with a $\mathrm{p}-\mathrm{GeSe}$ on $\mathrm{SiO}_{2} / \mathrm{Si}$ substrate. The optical microscope image of the device is illustrated in Fig. 1b. The detail transfer flakes process is provided in experimental detail. Atomic force microscopy (AFM) was used to evaluate the thickness of p-GeSe $(\sim 40 \mathrm{~nm})$ presented in Fig. S1. Raman spectrum of GeSe nanoflake was depicted in Fig. S1. It has been stated that the metals having lower work function are suitable for $\mathrm{p}-\mathrm{GeSe}$ to form a facile Schottky barrier height ${ }^{5,43,44}$. It was found that $\mathrm{p}$-GeSe and metal interface could be either an ohmic or rectifying behavior depending on metals and semiconductor working function value. Figure $1 c$ depicts $\mathrm{GeSe}(\Phi \sim 4.83 \mathrm{eV})^{47}, \mathrm{Pd}(\Phi \sim 5.6 \mathrm{eV})^{43}$ and $\mathrm{Cr}(\Phi \sim 4.5 \mathrm{eV})^{5,48}$ were used as electrode contacts in GeSe flake. Initially, the electrical characteristics of the p-GeSe FETs with Cr/Au and Pd/ $\mathrm{Au}$ electrodes were measured. The transfer characteristics show typical p-type semiconductor characteristics, depicted in Fig. 1(d,e). The field-effect carrier mobility $\left(\mu_{\mathrm{FE}}\right)$ was calculated for individual metal contacts using the following equation:

$$
\mu_{F E}=\frac{\mathrm{L}}{\mathrm{W}}\left(\frac{\mathrm{dI}_{\mathrm{ds}}}{\mathrm{dV}_{\mathrm{bg}}}\right) \frac{1}{\mathrm{C}_{\mathrm{bg}} \mathrm{V}_{\mathrm{ds}}}
$$

where $\mathrm{W}$ and $\mathrm{L}$ are the channel width and length, respectively, $\mathrm{C}_{\mathrm{bg}}$ is the gate capacitance $\left(\sim 115 \mathrm{aF} / \mu \mathrm{m}^{2}\right)$ for the $300 \mathrm{~nm}$-thick $\mathrm{SiO}_{2} / \mathrm{Si}$ substrates and $\left(\frac{\mathrm{dI}_{\mathrm{ds}}}{\mathrm{dV}_{\mathrm{bg}}}\right)$ is the slope of the transfer curve. The hole mobility of GeSe with $\mathrm{Cr} /$ Au contacts was measured to be $1.98 \mathrm{~cm}^{2} \mathrm{~V}^{-1} \mathrm{~s}^{-1}$ with a current on/off ratio of $\sim 10^{2}$, while the mobility of GeSe with $\mathrm{Pd} / \mathrm{Au}$ electrodes was estimated to be $5.02 \mathrm{~cm}^{2} \mathrm{~V}^{-1} \mathrm{~s}^{-1}$ with $\sim 10^{4}$. Our findings revealed that the mobility of $\mathrm{GeSe}$ with $\mathrm{Cr} / \mathrm{Au}$ decreased than that of one with $\mathrm{Pd} / \mathrm{Au}$ because of the Schottky junction formed at both $\mathrm{Cr} / \mathrm{Au}$ contacts, which resist to the follow of charges. In the $\mathrm{Pd} / \mathrm{Au}$ configuration, both contacts were formed with ohmic behavior because of the high work function of $\mathrm{Pd}(\Phi \sim 5.6 \mathrm{eV})^{49}$ further, the gate voltage $\left(\mathrm{V}_{\mathrm{bg}}\right)$ dependent $\mathrm{I}_{\mathrm{D}}-\mathrm{V}_{\mathrm{D}}$ output characteristics shows rectification behavior for the $\mathrm{Cr} / \mathrm{Au}$ metal contacts because of the formation of the Schottky barrier with P-GeSe as depicted in Fig. 1f. From the linear characteristics of $I-V$ output, we could predict that ohmic junction was formed with Pd/Au contacts, as shown in Fig. 1g.

The rectification of $\mathrm{p}$-GeSe with asymmetric contacts of $\mathrm{Pd} / \mathrm{Au}$ and $\mathrm{Cr} / \mathrm{Au}$ explicitly showed gate-dependent rectification, especially high rectifying behavior at a negative gate voltage due to higher Schottky barrier height between $\mathrm{Cr}$ and $\mathrm{GeSe}$, as illustrated in $\mathrm{V}$. When the forward bias voltage is applied, the rectifying current increases because of decreased barrier height. A rectification ratio (defined as the ratio of reverse current $\mathrm{I}_{\mathrm{r}}$ and forward current $\mathrm{I}_{\mathrm{f}}$ ) is obtained $10^{3}$ as depicted in Fig. $2 \mathrm{c}$, which is consistent with the other devices shown in Fig. S3.

We estimated the ideality factor to confirm the good performance of rectifying behavior of SBD by using thermionic emission theory. According to this theory, the current $\mathrm{I}_{\mathrm{D}}$ through a metal-semiconductor junction is given by ${ }^{50,51}$

$$
I_{D}=I_{S}\left[\exp \left(\frac{q V}{n K_{B} T}\right)-1\right]
$$

where $I_{S}$ is the reverse bias saturation current, $n$ is an ideality factor, q is an elementary charge, T is the temperature, and $K_{B}$ is the Boltzmann's constant. After interpretation above equation becomes 
(a)
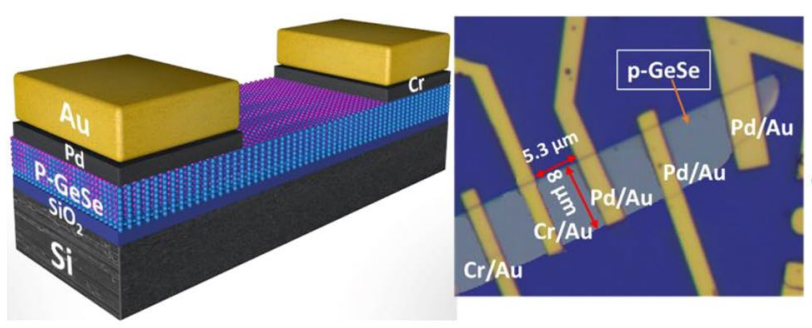

(c)

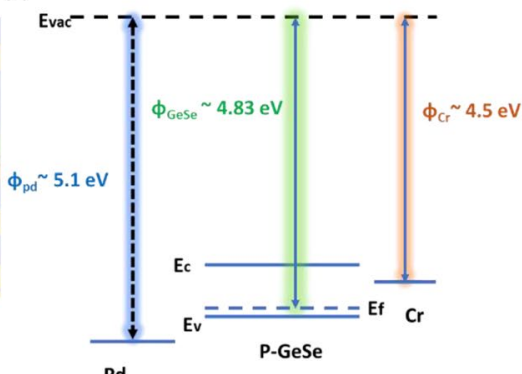

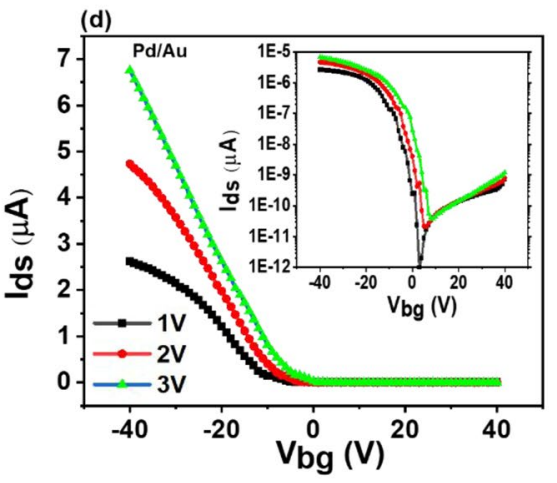
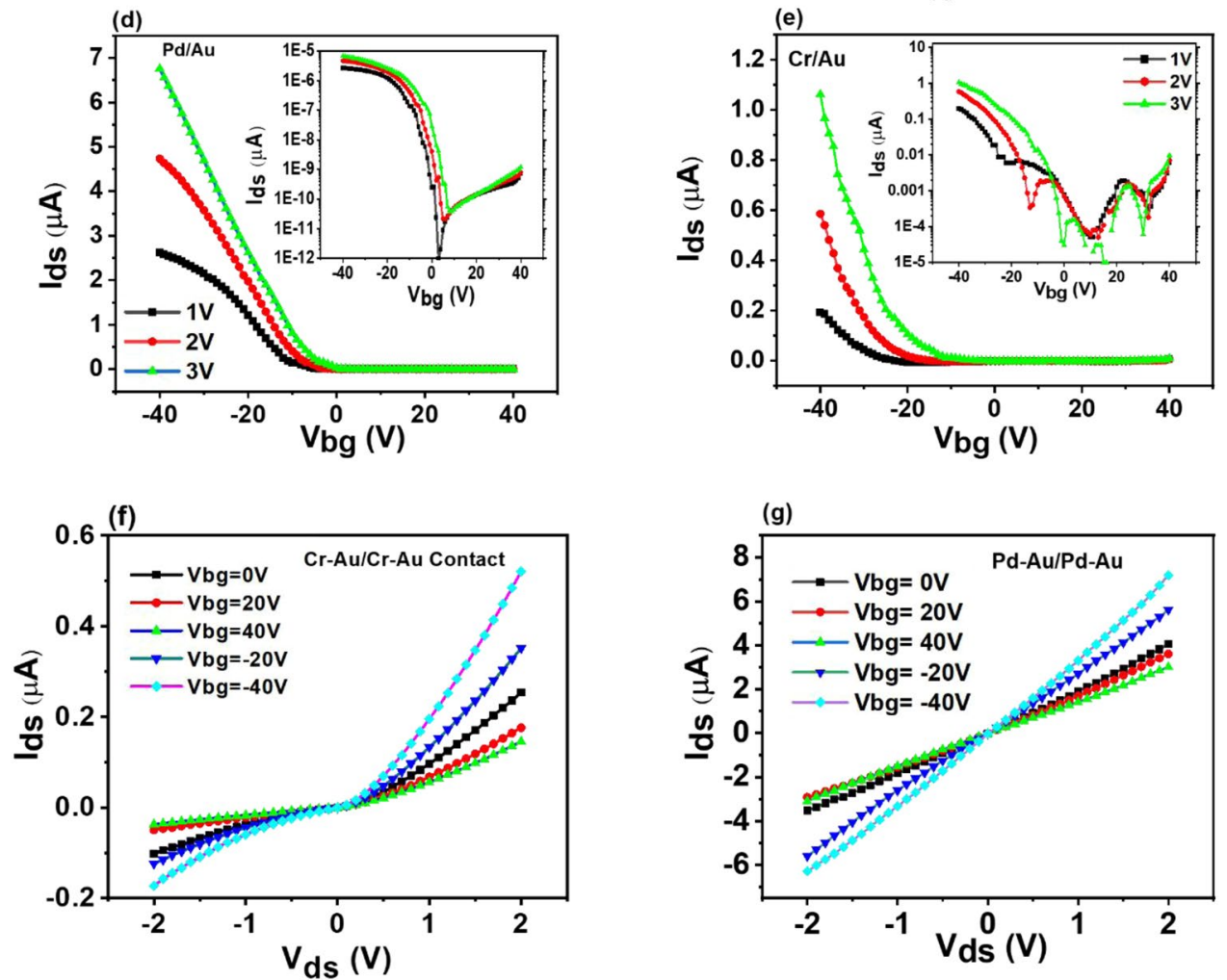

Figure 1. (a) Schematic view of p-GeSe Schottky diode. (b) Optical microscope image of the device. (c) Band diagram of Pd/p-GeSe/Cr. $I_{D^{-}} V_{G}$ characteristics of (d) p-GeSe FET with Pd/Au and (e) Cr/Au with $\mathrm{V}_{\mathrm{ds}}=1 \mathrm{~V}$ to 3 V.T.the semi-logarithmic plots of transfer characteristics are depicted in the insets on each figure. $I_{D}-V_{D}$ characteristics as a function of gate bias (f) Schottky contacts effect of $\mathrm{p}-\mathrm{GeSe}$ with $\mathrm{Cr} / \mathrm{Au}$. (g) Ohmic contact behavior with $\mathrm{Pd} / \mathrm{Au}$.

$$
\ln \left(I_{D}\right)=\ln \left(I_{S}\right)+\left(\frac{q}{n K_{B} T}\right) V
$$

The ideality factor (n) can be obtained via the following equation:

$$
n=\left(\frac{q}{K_{B} T}\right)\left(\frac{\mathrm{d} V}{\mathrm{~d} \ln I_{D}}\right)
$$

The lowest value of the ideality factor $(\mathrm{n})$ we evaluated was $\sim 1.8$. To extract the Schottky barrier height $(\mathrm{SBH})$ of $\operatorname{Pd}(\phi=5.6 \mathrm{eV})$ and $\mathrm{Cr}(\phi=4.5 \mathrm{eV})$, respectively, we measured temperature-dependent transfer characteristics as shown in Fig. S2.

According to thermionic emission theory, we used the following equation ${ }^{5,52-54}$

$$
\mathrm{I}_{\mathrm{ds}}=A_{\text {device }} A^{*} \mathrm{~T}^{2} \exp \left[-\frac{\mathrm{q}}{\mathrm{k}_{\mathrm{B}} \mathrm{T}}\left(\Phi_{\mathrm{B}}-\frac{\mathrm{V}_{\mathrm{ds}}}{\mathrm{n}}\right)\right]
$$

where $A_{\text {device }}, A^{*}$ are the area of the detector and Richardson constant, $\mathrm{q}$ is the elementary charge of the electron, $\mathrm{T}$ is the temperature $\mathrm{K}_{\mathrm{B}}$ is Boltzmann's constant, $\mathrm{V}_{\mathrm{ds}}$ the source-drain voltage, and $\mathrm{n}$ is the ideality factor. We drew 

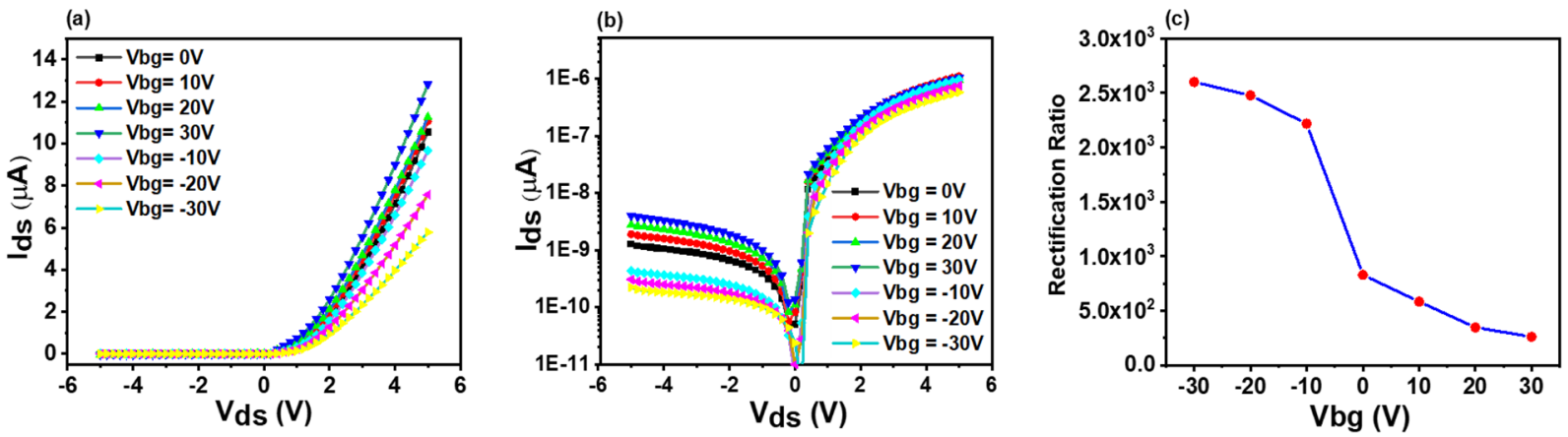

Figure 2. (a) $I-V$ characteristics of $\mathrm{p}-\mathrm{GeSe} S$ chottky junction between $\mathrm{Cr} / \mathrm{Au}-\mathrm{Pd} / \mathrm{Au}$ contacts at different gate voltages. (b) the semi-logarithmic plots of output characteristics (c) Gate dependent rectification ratio of p-GeSe Schottky junction.
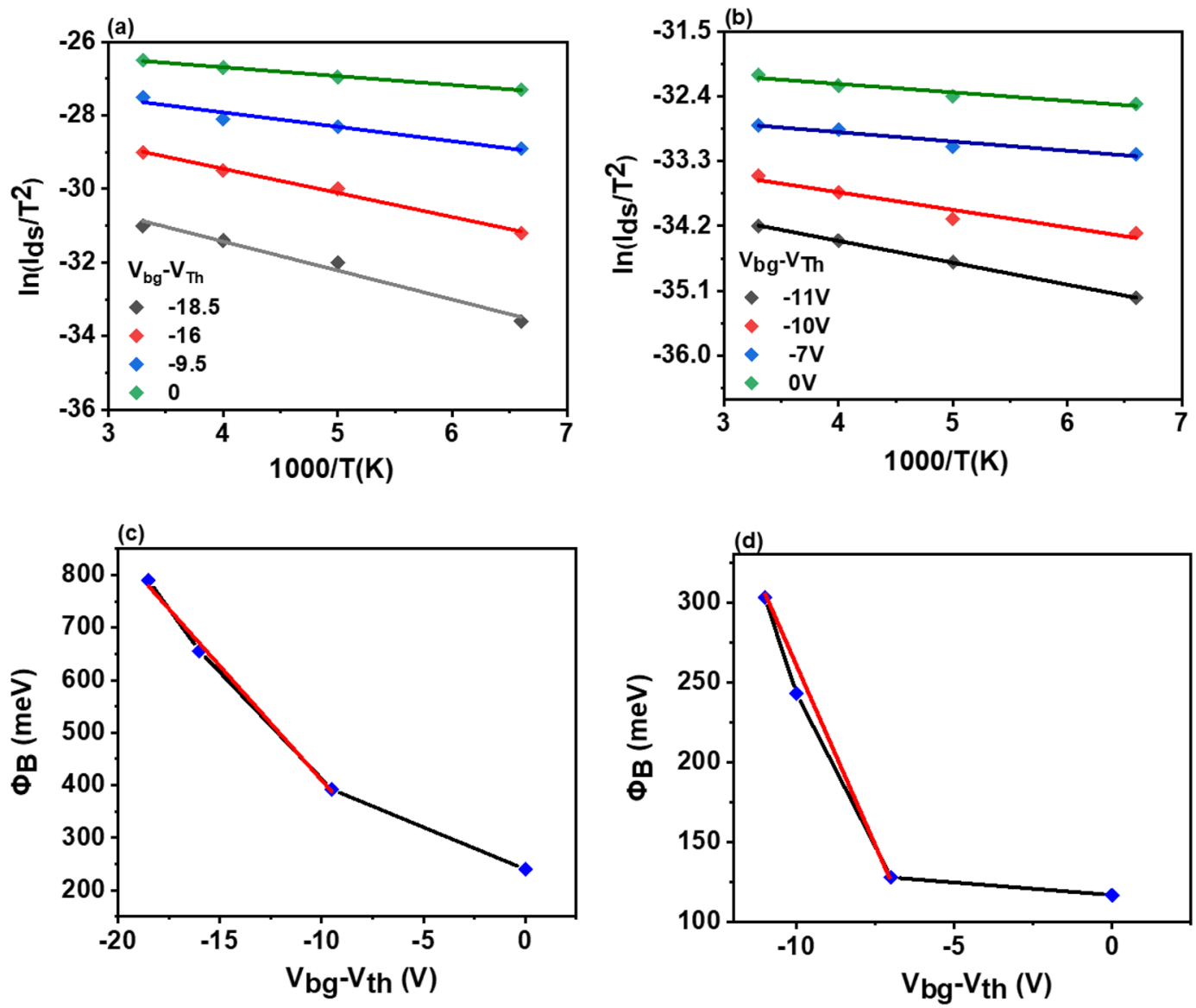

Figure 3. (a) Typical Richardson's plot $\ln \left(\mathrm{I}_{\mathrm{ds}} / \mathrm{T}^{2}\right)$ versus 1000/T for (a) Cr/Au and (b) for Pd/Au metal contacts. Corresponding Schottky barrier Potential (c) at the p-GeSe/ Cr/Au and (d) Pd/Au junction as a function of Vbg-Vth.

the Arrhenius plot of the device at different gate voltages for both metals Pd and Cr shown in Fig. 3a,b, respectively. We estimated the $\mathrm{SBH}$ for $\mathrm{Pd}$ and $\mathrm{Cr}$ with p-GeSe by taking the slope of $\ln \left(\mathrm{I}_{\mathrm{ds}} / \mathrm{T}^{2}\right)$ versus $1000 / \mathrm{T}$, as shown in Fig. 3c,d. We found the SBH of Cr with p-GeSe is much higher than SBH of Pd because of ohmic behavior between Pd and p-GeSe.

Moreover, we also extensively studied self-powered photoresponse characteristics of SBD. Self-power photodetectors are devices in which photogenerated charge carriers are separated by the built-in potential and there is no requirement of any external power source. $\mathrm{Pd} / \mathrm{Au}-\mathrm{GeSe}-\mathrm{Pd} / \mathrm{Au}$ structure does not show any photoresponse at zero bias since it has both ohmic contacts. However, $\mathrm{Cr} / \mathrm{Au}-\mathrm{GeSe}-\mathrm{Cr} / \mathrm{Au}$ form Schottky contacts, which may show a photoresponse at zero bias. But the symmetric contact electrodes may cause a net null current due to equal but opposite directions of photogenerated carriers. Hence, we designed a dual junction photodetector 

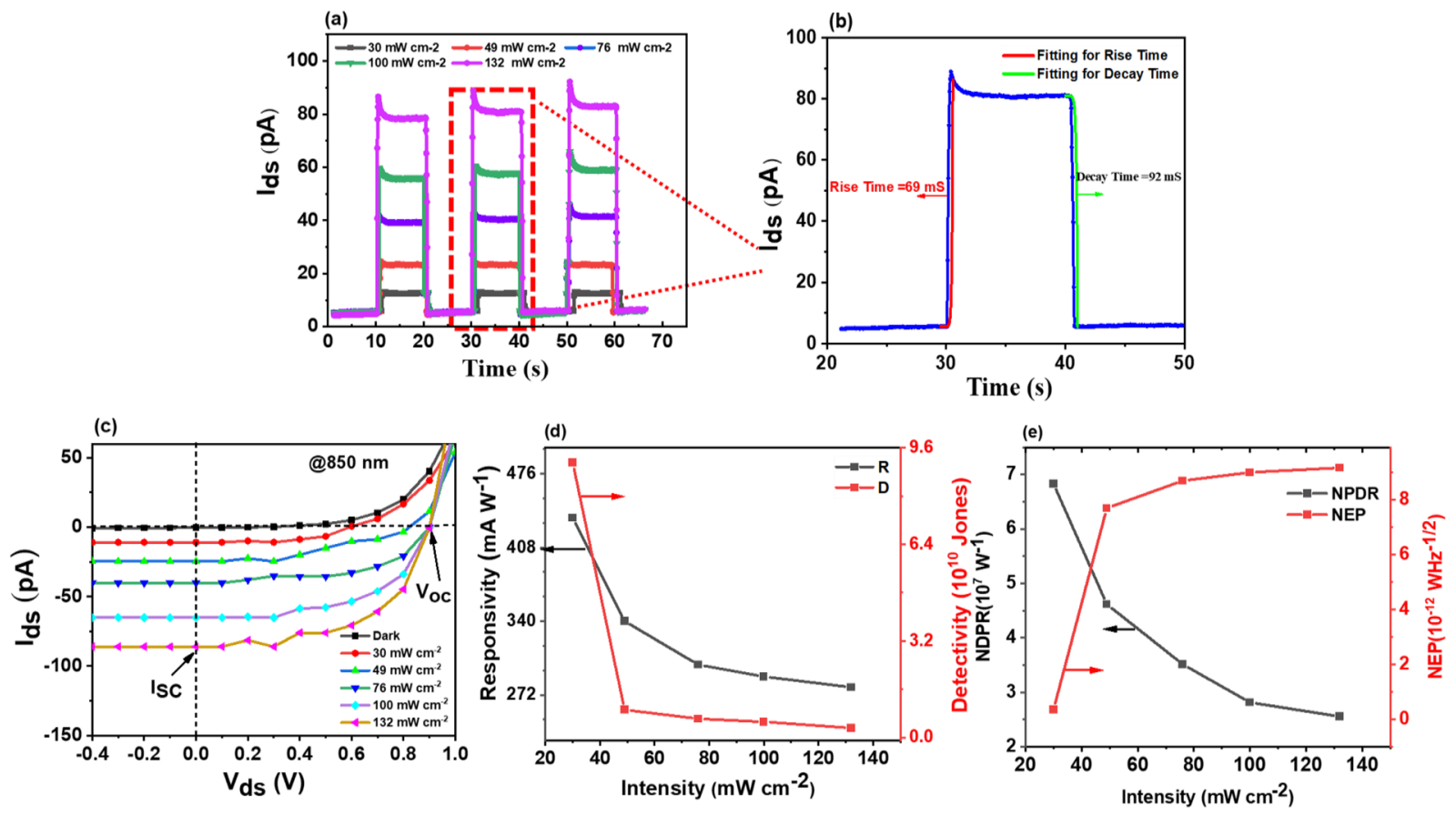

Figure 4. (a) Time dependent photoresponse of p-GeSe Schottky junction under illuminations with different laser light (@850 nm) intensity at $\mathrm{V}_{\mathrm{ds}}=0 \mathrm{~V}$. (b) rise time and decay time (c) $I-V$ characteristics of $\mathrm{p}-\mathrm{GeSe}$ Schottky junction under dark and variable intensities (d) responsivity, $\mathrm{R}\left(\mathrm{mA} \mathrm{W}^{-1}\right)$, and detectivity, $\mathrm{D}$ (Jones) with the variation of light intensities (e) normalized photocurrent to dark current ratio NPDR $\left(\mathrm{W}^{-1}\right)$ and noise equivalent power $\mathrm{NEP}\left(\mathrm{W} \mathrm{Hz}^{-1 / 2}\right)$ with varying light intensities.

with asymmetric contacts electrodes having a configuration of $\mathrm{Pd} / \mathrm{Au}-\mathrm{GeSe}-\mathrm{Cr} / \mathrm{Au}$. This photodetector uses the built-in potential present in metal contact and p-GeSe junction interface (forming Schottky junction) to separate and fast transfer of photogenerated electron-hole pairs, which empowers them to detect light signals without an external bias voltage.

Figure S4 illustrates the wavelength-dependent photovoltaic behavior under irradiation of light with wavelength $(\lambda)$ of $220 \mathrm{~nm}, 530 \mathrm{~nm}$, and $850 \mathrm{~nm}$ at zero voltage bias in p-GeSe Schottky diode, as illumination wavelength decreased, the reverse and forward bias currents drastically increased. This implies that photoexcited electron-hole pairs significantly increases with greater photon energy ${ }^{55-57}$. Figure S4a shows the output characteristics of the device under the illumination of light having wavelengths $(\lambda) 220 \mathrm{~nm}, 530 \mathrm{~nm}$ and $850 \mathrm{~nm}$, respectively, and Fig. S4(b-d) depicts the transient dynamic photoresponse. Figure $4 \mathrm{c}$ shows the $I-V$ characteristics of the device under the illumination of near-infrared radiation (NIR) with a wavelength of $850 \mathrm{~nm}$. The linear plot explicitly shows that in the presence of photogenerated current at the zero-bias device can be a self-powered photodetector under the action of open-circuit voltage $\left(\mathrm{V}_{\mathrm{oc}}\right)$. Furthermore, with irradiation of various intensities ranging from 30 to $132 \mathrm{~mW} \mathrm{~cm}^{-2}$, the device clearly shows systematic deviation from the dark level. We observed open-circuit voltage $\left(\mathrm{V}_{\mathrm{oc}}\right)$ of $0.9 \mathrm{~V}$ and short-circuit current $\left(\mathrm{I}_{\mathrm{sc}}\right)$ of $80 \mathrm{pA}$ for the $132 \mathrm{~mW} \mathrm{~cm} \mathrm{~m}^{-2}$ light intensity. The highest values of $\mathrm{V}_{\mathrm{oc}}$ and $\mathrm{I}_{\mathrm{sc}}$ were obtained with a power intensity of $132 \mathrm{~mW} \mathrm{~cm}^{-2}$ due to excessive photogenerated charge carriers under the effect of abundant photons in the high intensity of light.

The transient photoresponse is a very critical parameter for a photodetector to be used in emergency applications such as fire detection, night vision, etc. Therefore, the dynamic photoresponse rise and fall time of the p-GeSe SBD was observed under NIR laser light irradiation with a wavelength $(\lambda) 850 \mathrm{~nm}$. The rise time $\left(\tau_{\mathrm{r}}\right.$, time taken by device to reach $90 \%$ from $10 \%$ ) and fall time ( $\tau_{\text {f }}$, time taken by device to decay from $90 \%$ to $\left.10 \%\right)^{58}$ of the p-GeSe Schottky barrier diode shows values of 69 and 92 ms, respectively (shown in Fig. 4b).

To further validate the gate tunable self-powered photovoltaic effect, we characterized the time-dependent photoresponse at various gate voltages. We realized that, without an external bias voltage and illuminating environment, the device reached its thermal equilibrium state when the Fermi levels of both metal and GeSe are aligned, resulting in the formation of a Schottky barrier (ohmic behavior) at $\mathrm{Cr}(\mathrm{Pd})$ and GeSe interface. When applying a back-gate voltage of $\mathrm{Vg}>0$, the electrons are attracted to the interface between $\mathrm{p}-\mathrm{GeSe}$ and $\mathrm{SiO} 2$ to form accumulation layer, the Fermi level of p-GeSe shifts upward that attributed to electrostatic doping of electrons, results in a larger potential hole barrier between the $\mathrm{p}-\mathrm{GeSe}$ and $\mathrm{Cr}$. Consequently, a built-in potential at $\mathrm{Cr}-\mathrm{GeSe}$ interface increased, this selective barrier at the junction is the means of separating charges during electron-holes generation under illumination ${ }^{18,59}$. It is the key to the production of a photovoltaic electric current ${ }^{60-62}$. Therefore, upon illumination of NIR wavelength of $850 \mathrm{~nm}$ with a power of $76 \mathrm{mWcm}^{-2}$ yielding the greater photovoltaic current with an increase of $\mathrm{Vg}$ from $0 \mathrm{~V}$ to $40 \mathrm{~V}$ without external power. In contrast, at $V g<0$ the Fermi level of p-GeSe moves downward, yielding smaller the hole potential barrier, results in smaller photovoltaic current under the illumination of the light of the same condition, shown in Fig. S5(a). Additionally, to 
investigate the stability of the photovoltaic performance of the device, we measured two more p-GeSe Schottky photodetector devices. All devices showed same reproducibility which is consistent with previous device measured results shown in Fig. S5(c).

The other vital figures of merit, for instance, responsivity, detectivity, normalized photocurrent to dark current ratio, and noise equivalent power are calculated with the variation of light power intensities. The responsivity $\left(\mathrm{R}=J_{p} / P_{i n}\right.$, where $J_{\mathrm{p}}$ is photocurrent density and $\mathrm{P}_{\text {in }}$ is input power per area) and detectivity $\left(D=R / \sqrt{2 J_{d}}\right.$, where $\mathrm{q}$ is elementary charge and $\mathrm{J}_{\mathrm{d}}$ is dark current density) are important aspects of photodetector ${ }^{63-65}$ shown in Fig. $4 \mathrm{~d}$. The greater value of responsivity is caused by the higher photocurrent ${ }^{58,63}$. Similarly, the device having a lower dark current provides the higher detectivity. Thus, the greater values of both $\mathrm{R}$ and $\mathrm{D}$ are an important feature of an efficient photodetector ${ }^{67,58,66}$. Our photodetector, based on $\mathrm{p}-\mathrm{GeSe}$ SBD, showed a high responsivity of 280 $\mathrm{mAW}^{-1}$ and a detectivity of $4.1 \times 10^{9}$ jones with a power intensity of $132 \mathrm{~mW} \mathrm{~cm}^{-2}$. Figure $4 \mathrm{e}$ shows the normalized photocurrent to dark current ratio $\left(\mathrm{NPDR}=\mathrm{R} / \mathrm{I}_{\mathrm{d}}\right.$, where $\mathrm{R}$ is responsivity and $\mathrm{I}_{\mathrm{d}}$ is the dark current) and noise equivalent power $\left(\mathrm{NEP}=1 /\left(\mathrm{NPDR} \sqrt{2 q / I_{d}}\right)\right)$. The NPDR of $3 \times 10^{7} \mathrm{w}^{-1}$ and NEP of $9.1 \times 10^{-12} \mathrm{WHz}^{-1 / 2}$ were investigated at a power intensity of $132 \mathrm{~mW} \mathrm{~cm}^{-2}$. The NEP revealed that the photodetector based on $\mathrm{p}-\mathrm{GeSe}$ Schottky barrier diode is capable of detecting power as low as picowatt, which is very crucial for emergency applications.

\section{Conclusion}

A self-powered photodetector was designed using asymmetrical metals contacts on a $2 \mathrm{D}$ semiconductor (p-GeSe). It has demonstrated outstanding gate dependent rectifying behavior with an excellent on/off ratio (up to $10^{3}$ ). In addition, stable rectifying behavior was also obtained with a built-in potential at the junction. This interfacial potential governs the separation of the photogenerated charge carriers making the fabricated device self-powered for optoelectronics applications. It has also exhibited a high rise and fall time of 69 and $92 \mathrm{~ms}$, respectively. Moreover, promising figures of merits were obtained, such as responsivity, detectivity, normalize photocurrent to dark current ratio and noise equivalent power of $280 \mathrm{mAW}^{-1}$, of $4.1 \times 10^{9} \mathrm{Jones}^{3} \times 10^{7} \mathrm{~W}^{-1}$ and $9.1 \times 10^{-12} \mathrm{WHz}^{-1 / 2}$ respectively. The NEP revealed that the photodetector based on $\mathrm{p}-\mathrm{GeSe}$ Schottky barrier diode is capable of detecting power as low as picowatt, which is very crucial for emergency applications.

\section{Methods}

Atomically thin flakes of $\mathrm{p}-\mathrm{GeSe}$ were peeled from their parent bulk crystals using a scotch tape mechanical exfoliation technique, similar to that employed for the exfoliation of graphene ${ }^{2,23}$ and transferred onto $\mathrm{Si} /$ $\mathrm{SiO}_{2}(300 \mathrm{~nm})$ substrate using transparent poly (dimethyl siloxane) (PDMS) stamp by aligned dry transfer ${ }^{24,68}$. Multilayer $\mathrm{p}$-GeSe nanoflakes were identified by optical microscope, nanoflakes and were characterized by Raman spectroscopy and the thickness of $\mathrm{p}-\mathrm{GeSe}(\sim 40 \mathrm{~nm})$ approximately equal to 40 layers evaluated by using atomic force microscopy (AFM). Electrodes were printed using electron beam lithography and $\mathrm{Pd} / \mathrm{Au}(10 / 20 \mathrm{~nm})$ and $\mathrm{Cr} / \mathrm{Au}(10 / 20 \mathrm{~nm})$ was evaporated and liftoff were carried out on multilayer p-GeSe nanoflakes. The electrical transport measurements at room temperature were characterized by using Keithley 4200A-SCS Parameter Analyzer. The photoresponse of SBD photodetector was measured using a continuous wave laser beam from the diode laser (from $220 \mathrm{~nm}$ to $850 \mathrm{~nm}$ ) directly illuminated onto the device.

Received: 26 February 2020; Accepted: 21 April 2020;

Published online: 10 June 2020

\section{References}

1. Novoselov, K. S. et al. Electric Field Effect in Atomically Thin Carbon Films. Science (80-.). 306, 666 LP-669 (2004)

2. Novoselov, K. S. et al. Two-dimensional atomic crystals. PNAS 102, 10451-10453 (2005).

3. Feng, W., Guo, G.-Y. \& Yao, Y. \%J 2D M. Tunable magneto-optical effects in hole-doped group-IIIA metal-monochalcogenide monolayers. 2D Mater. 4, 15017 (2016).

4. Pati, S. K., Enoki, T. \& rao. GRAPHENE AND ITS FASCINATING ATTRIBUTES. https://doi.org/10.1142/9789814329361 (World Scientific Publishing Co. Pte. Ltd., 2011)

5. Aftab, S. et al. Formation of an MoTe2 based Schottky junction employing ultra-low and high resistive metal contacts. RSC Adv. 9, 10017-10023 (2019).

6. Radisavljevic, B., Radenovic, A., Brivio, J., Giacometti, V. \& Kis, A. Single-layer MoS2 transistors. Nat. Nanotechnol. 6, 147-150 (2011).

7. Georgiou, T. et al. Vertical field-effect transistor based on graphene-WS 2 heterostructures for flexible and transparent electronics. 8, 100 (2013).

8. Late, D. J. et al. GaS and GaSe ultrathin layer transistors. Adv. Mater. 24, 3549-3554 (2012).

9. Lei, S. et al. Evolution of the electronic band structure and efficient photo-detection in atomic layers of InSe. ACS Nano 8, $1263-1272$ (2014).

10. Song, X., Hu, J. \& Zeng, H. Two-dimensional semiconductors: recent progress and future perspectives. J. Mater. Chem. C 1, 2952-2969 (2013).

11. Hu, P., Wen, Z., Wang, L., Tan, P. \& Xiao, K. \%J A. C. S. nano. Synthesis of few-layer GaSe nanosheets for high performance photodetectors. ASC Nano 6, 5988-5994 (2012).

12. Xie, J. et al. Controllable Disorder Engineering in Oxygen-Incorporated MoS2 Ultrathin Nanosheets for Efficient Hydrogen Evolution. J. Am. Chem. Soc. 135, 17881-17888 (2013).

13. Li, X. et al. Controlled vapor phase growth of single crystalline, two-dimensional GaSe crystals with high photoresponse. Sci. Rep. 4 , 5497 (2014).

14. Zhou, Y. et al. Epitaxy and photoresponse of two-dimensional GaSe crystals on flexible transparent mica sheets. ASC Nano 8, 1485-1490 (2014).

15. Yang, S. et al. Highly efficient gas molecule-tunable few-layer GaSe phototransistors. J. Mater. Chem. C 4, 248-253 (2016).

16. Hu, P. et al. Highly sensitive phototransistors based on two-dimensional GaTe nanosheets with direct bandgap. Nano Res. 7, 694-703 (2014).

17. Liu, F. et al. High-sensitivity photodetectors based on multilayer GaTe flakes. ACS Nano 8, 752-760 (2014). 
18. Tamalampudi, S. R. et al. High performance and bendable few-layered InSe photodetectors with broad spectral response. Nano Lett. 14, 2800-2806 (2014).

19. Lei, S. et al. An atomically layered InSe avalanche photodetector. Nano Lett. 15, 3048-3055 (2015).

20. Sucharitakul, S. et al. Intrinsic electron mobility exceeding $103 \mathrm{~cm} 2 /(\mathrm{V} \mathrm{s})$ in multilayer InSe FETs. Nano Lett. 15, 3815-3819 (2015).

21. Chen, Z., Biscaras, J. \& Shukla, A. \%J N. A high performance graphene/few-layer InSe photo-detector. Nanoscale 7, 5981-5986 (2015)

22. Dean, C. R. et al. Boron nitride substrates for high-quality graphene electronics. Nat. Nanotechnol. 5, 722-726 (2010).

23. Mukherjee, B. et al. NIR Schottky photodetectors based on individual single-crystalline GeSe nanosheet. ACS Appl. Mater. Interfaces 5, 9594-9604 (2013).

24. Yap, W. C. et al. Layered material GeSe and vertical GeSe/MoS2 p-n heterojunctions. Nano Res. 11, 420-430 (2018).

25. Makinistian, L. \& Albanesi, E. A. Ab initiocalculations of the electronic and optical properties of germanium selenide. J. Phys. Condens. Matter 19, 186211 (2007).

26. Singh, A. K. \& Hennig, R. G. \% A. P. L. Computational prediction of two-dimensional group-IV mono-chalcogenides. Appl. Phys. Lett. 105, 42103 (2014)

27. Gomes, L. C., Trevisanutto, P. E., Carvalho, A., Rodin, A. S. \& Neto, A. H. C. \%J P. R. B. Strongly bound Mott-Wannier excitons in $\mathrm{GeS}$ and GeSe monolayers. Phys. Rev. B 94, 155428 (2016).

28. Chamlagain, B. et al. Mobility improvement and temperature dependence in MoSe2 field-effect transistors on parylene-C substrate. ASC Nano 8, 5079-5088 (2014).

29. Liu, S.-C., Yang, Y., Li, Z., Xue, D.-J. \& Hu, J.-S. GeSe thin-film solar cells. Mater. Chem. Front. 4, 775-787 (2020).

30. Koike, M. et al. Dynamical Spin Injection into p-Type Germanium at Room Temperature. Appl. Phys. Express 6, 23001 (2013).

31. Afzal, A. M., Iqbal, M. Z., Mumtaz, S. \& Akhtar, I. Multifunctional and high-performance GeSe/PdSe2 heterostructure device with a fast photoresponse. J. Mater. Chem. C 8, 4743-4753 (2020).

32. Asanabe, S. \& Okazaki, A. \%J J. of the P. S. of J. Electrical properties of germanium selenide GeSe. J. Phys. Soc. Japan 15, 989-997 (1960).

33. Kallatt, S., Umesh, G., Bhat, N. \& Majumdar, K. Photoresponse of atomically thin MoS2 layers and their planar heterojunctions. Nanoscale 8, 15213-15222 (2016).

34. Buscema, M. et al. Large and Tunable Photothermoelectric Effect in Single-Layer MoS2. Nano Lett. 13, 358-363 (2013).

35. Abbas, S. et al. Transparent and Flexible In2O3 Thin Film for Multilevel Nonvolatile Photomemory Programmed by Light. ACS Appl. Electron. Mater. 1, 437-443 (2019).

36. Li, X. et al. Two-dimensional GaSe/MoSe\&lt;sub\&gt;2\&lt;/sub\&gt; misfit bilayer heterojunctions by van der Waals epitaxy. Sci. Adv. 2, e1501882 (2016)

37. Ye, L., Li, H., Chen, Z. \& Xu, J. Near-Infrared Photodetector Based on MoS2/Black Phosphorus Heterojunction. ACS Photonics 3 , 692-699 (2016)

38. Serrano, W., Pinto, N. J., Naylor, C. H., Kybert, N. J. \& Johnson, A. T. C. Facile fabrication of a ultraviolet tunable MoS2/p-Si junction diode. Appl. Phys. Lett. 106, 193504 (2015).

39. Cheng, R. et al. Electroluminescence and Photocurrent Generation from Atomically Sharp WSe2/MoS2 Heterojunction $p-n$ Diodes. Nano Lett. 14, 5590-5597 (2014).

40. Jin, Y. et al. A Van Der Waals Homojunction: Ideal p-n Diode Behavior in MoSe2. Adv. Mater. 27, 5534-5540 (2015).

41. Mehboudi, M. et al. Two-dimensional disorder in black phosphorus and monochalcogenide monolayers. Nano Lett. 16, 1704-1712 (2016).

42. Yang, M. H., Teo, K. B. K., Milne, W. I. \& Hasko, D. G. \%J A. P. L. Carbon nanotube Schottky diode and directionally dependent field-effect transistor using asymmetrical contacts. Appl. Phys. Lett. 87, 253116 (2005).

43. Hughes, M. A. et al. \%J A. P. L. An ultra-low leakage current single carbon nanotube diode with split-gate and asymmetric contact geometry. Appl. Phys. Lett. 103, 133508 (2013).

44. Ryu, B. et al. Photostable dynamic rectification of one-dimensional schottky diode circuits with a zno nanowire doped by $\mathrm{H}$ during passivation. Nano Lett. 11, 4246-4250 (2011).

45. K, K. N. Front Matter. Complete Guide to Semiconductor Devices, https://doi.org/10.1002/9781118014769.fmatter (2009).

46. Zhao, Y. et al. Influence of Asymmetric Contact Form on Contact Resistance and Schottky Barrier, and Corresponding Applications of Diode. ACS Appl. Mater. Interfaces 9, 18945-18955 (2017).

47. Xue, D.-J. et al. GeSe Thin-Film Solar Cells Fabricated by Self-Regulated Rapid Thermal Sublimation. J. Am. Chem. Soc. 139, 958-965 (2017)

48. Dhar, N. et al. Effect Of N-Type Transition Metal Dichalcogenide Molybdenum Telluride (N-Mote2) In Back Contact. Interface Of Cadmium Telluride Solar Cells From Numerical Analysis Chalcogenide Lett. 11, (2014).

49. Kim, C. et al. Fermi level pinning at electrical metal contacts of monolayer molybdenum dichalcogenides. ACS Nano 11, 1588-1596 (2017).

50. Dai, M. et al. A dual-band multilayer InSe self-powered photodetector with high performance induced by surface plasmon resonance and asymmetric Schottky junction. ACS Nano 12, 8739-8747 (2018).

51. Lee, S., Tang, A., Aloni, S. \& Philip Wong, H.-S. Statistical Study on the Schottky Barrier Reduction of Tunneling Contacts to CVD Synthesized MoS2. Nano Lett. 16, 276-281 (2016).

52. Neamen, D. A. Semiconductor physics and devices. vol. 3 (McGraw-Hill New York, 1997)

53. Banwell, T. C. \& Jayakumar, A. \%J E. letters. Exact analytical solution for current flow through diode with series resistance. Electron. Lett. 36, 291-292 (2000).

54. Lee, J. H. et al. Photocurrent switching of monolayer MoS2 using a metal-insulator transition. Nano Lett. 17, 673-678 (2017).

55. Teng, F., Hu, K., Ouyang, W. \& Fang, X. Photoelectric Detectors Based on Inorganic p-Type Semiconductor Materials. Adv. Mater. 30, 1706262 (2018)

56. Chen, J., Ouyang, W., Yang, W., He, J.-H. \& Fang, X. Recent Progress of Heterojunction Ultraviolet Photodetectors: Materials, Integrations, and Applications. Adv. Funct. Mater. n/a, 1909909 (2020).

57. Zhang, Y. et al. Self-Powered Dual-Color UV-Green Photodetectors Based on SnO2 Millimeter Wire and Microwires/CsPbBr3 Particle Heterojunctions. J. Phys. Chem. Lett. 10, 836-841 (2019).

58. Abbas, S., Kumar, M. \& Kim, J. \% M M. S. In S. P. All metal oxide-based transparent and flexible photodetector. Mater. Sci. Semicond. Process. 88, 86-92 (2018).

59. Lopez-Sanchez, O., Lembke, D., Kayci, M., Radenovic, A. \& Kis, A. Ultrasensitive photodetectors based on monolayer MoS2. Nat. Nanotechnol. 8, 497-501 (2013).

60. Liu, H., Gao, N., Liao, M. \& Fang, X. Hexagonal-like Nb2O5 Nanoplates-Based Photodetectors and Photocatalyst with High Performances. Sci. Rep. 5, 7716 (2015).

61. Wu, E. et al. In Situ Fabrication of 2D WS2/Si Type-II Heterojunction for Self-Powered Broadband Photodetector with Response up to Mid-Infrared. ACS Photonics 6, 565-572 (2019).

62. Wu, D. et al. Mixed-dimensional PdSe2/SiNWA heterostructure based photovoltaic detectors for self-driven, broadband photodetection, infrared imaging and humidity sensing. J. Mater. Chem. A 8, 3632-3642 (2020).

63. Abbas, S. et al. Silver-Nanowire-Embedded Transparent Metal-Oxide Heterojunction Schottky Photodetector. ACS Appl. Mater. Interfaces 10, 14292-14298 (2018) 
64. Xie, C. \& Yan, F. Flexible photodetectors based on novel functional materials. Small 13, 1701822 (2017).

65. Monroy, E., Omnès, F. \& Calle, F. Wide-bandgap semiconductor ultraviolet photodetectors. Semicond. Sci. Technol. 18, R33 (2003).

66. Patel, M. \& Kim, J. Transparent NiO/ZnO heterojunction for ultra-performing zero-bias ultraviolet photodetector on plastic substrate. J. Alloys Compd. 729, 796-801 (2017).

67. Abbas, S. \& Kim, J. All-metal oxide transparent photodetector for broad responses. Sensors and Actuators, A: Physical vol. 303 (2020).

68. Castellanos-Gomez, A. et al. Deterministic transfer of two-dimensional materials by all-dry viscoelastic stamping. $2 D$ Mater. 1, 11002 (2014).

\section{Acknowledgements}

This research was supported by the Nano Material Technology Development Program through the National Research Foundation of Korea (NRF) funded by the Ministry of Science, ICT \& Future Planning (NRF2016M3A7B4909942) as well as 2016R1D1A1B01015047. This research was also sponsored by the Korea Institute of Energy Technology Evaluation and Planning and the Ministry of Trade, Industry and Energy of the Republic of Korea (20172010106080).

\section{Author contributions}

M.H., S.A., A.A., S.H.A.J.: Designed, carried out experiments. Analyzed data and co-wrote paper. N.D.C., Y.S.:Performed AFM experiments and analyzed data. P.G., H.N., J.E.: Performed low temperature measurements and analyzed data. S.H., R.A.: Co-wrote paper. J.J.: Designed experiments, analyzed data, and edited paper.

\section{Competing interests}

The authors declare no competing interests.

\section{Additional information}

Supplementary information is available for this paper at https://doi.org/10.1038/s41598-020-66263-8.

Correspondence and requests for materials should be addressed to J.J.

Reprints and permissions information is available at www.nature.com/reprints.

Publisher's note Springer Nature remains neutral with regard to jurisdictional claims in published maps and institutional affiliations.

(c) (i) Open Access This article is licensed under a Creative Commons Attribution 4.0 International License, which permits use, sharing, adaptation, distribution and reproduction in any medium or format, as long as you give appropriate credit to the original author(s) and the source, provide a link to the Creative Commons license, and indicate if changes were made. The images or other third party material in this article are included in the article's Creative Commons license, unless indicated otherwise in a credit line to the material. If material is not included in the article's Creative Commons license and your intended use is not permitted by statutory regulation or exceeds the permitted use, you will need to obtain permission directly from the copyright holder. To view a copy of this license, visit http://creativecommons.org/licenses/by/4.0/.

(C) The Author(s) 2020 